\title{
A generalized Poisson equation and short-range self-interaction energies
}

\author{
Sergey A. Varganov, Andrew T. B. Gilbert, and Peter M. W. Gill ${ }^{\text {a) }}$ \\ Research School of Chemistry, Australian National University, Canberra ACT 0200, Australia
}

(Received 18 February 2008; accepted 23 May 2008; published online 24 June 2008)

\begin{abstract}
We generalize the Poisson equation to attenuated Newtonian potentials. If the attenuation is at least exponential, the equation provides a local mapping between the density and its potential. We use this to derive several density functionals for the short-range self-interaction energy. (C) 2008 American Institute of Physics. [DOI: 10.1063/1.2945298]
\end{abstract}

\section{INTRODUCTION}

There has been a recent surge of interest ${ }^{1-20}$ in electronic structure methods that split the Coulomb operator,

$$
u^{-1} \equiv S(u)+L(u)
$$

(where $\boldsymbol{u}=\boldsymbol{r}-\boldsymbol{r}^{\prime}$ ) into short- and long-range components. The problem of computing the Coulomb potential of a density $\rho(\boldsymbol{r})$ then splits into the subproblems of computing its shortrange part

$$
V(\boldsymbol{r})=\int S(u) \rho\left(\boldsymbol{r}^{\prime}\right) d \boldsymbol{r}^{\prime}
$$

and the complementary long-range part. The latter can be treated by Fourier or fast multipole methods, but the shortrange potential $V(\boldsymbol{r})$ often presents a burdensome computational bottleneck. $^{21}$

In many of these methods, ${ }^{1,3,6-8,11,14,15,19,20} V(\boldsymbol{r})$ is found by embedding the generation of attenuated two-electron integrals within a complicated cutoff strategy. We have shown $^{21-23}$ how these integrals can be computed when $\rho(\boldsymbol{r})$ is expanded in a Gaussian basis, but if $S(u)$ is a short-ranged function, it is reasonable to ask whether integration over all space is appropriate and necessary. More specifically, one should ask whether there exists a local relationship between $V(\boldsymbol{r})$ and $\rho(\boldsymbol{r})$.

Newtonian potentials can be attenuated in many ways. We will focus here on the Yukawa attenuator ${ }^{24}$

$$
S_{Y}(u)=\frac{\exp (-\omega u)}{u}
$$

the Ewald attenuator $2,3,25,26$

$$
S_{E}(u)=\frac{\operatorname{erfc}(\omega u)}{u},
$$

and the Heaviside attenuator

$$
S_{H}(u)= \begin{cases}1 / u & \text { if } \omega u \leqslant 1 \\ 0 & \text { if } \omega u>1\end{cases}
$$

where the parameter $\omega$ controls the attenuation strength. The Fermi-Dirac, Chakravorty and Clementi, ${ }^{27}$ Overhauser, ${ }^{28}$

\footnotetext{
${ }^{a)}$ Electronic mail: peter.gill@anu.edu.au.
}

and "optimal" 29 attenuators are also interesting, and our approach below is equally amenable to these.

In this Communication, we show that the short-range potential $V(\boldsymbol{r})$ of a smooth density $\rho(\boldsymbol{r})$ is governed by a generalized Poisson equation (GPE) and if $S(u)$ decays at least exponentially, $V(\boldsymbol{r})$ is locally related to $\rho(\boldsymbol{r})$. We then illustrate this by constructing several density functionals for the short-range self-interaction energy. These can be exploited in any field where Newtonian potentials arise, including electronic structure, molecular dynamics, classical electrostatics, fluid mechanics, and cosmology.

\section{GENERALIZED POISSON EQUATION}

By the convolution theorem, the short-range potential [Eq. (2)] can be expressed as

$$
V(\boldsymbol{r})=4 \pi \int \sigma(k) \hat{\rho}(\boldsymbol{k}) e^{i \boldsymbol{k} \cdot \boldsymbol{r}} d \boldsymbol{k},
$$

where

$$
\begin{aligned}
& \sigma(k)=\int_{0}^{\infty} u^{2} j_{0}(k u) S(u) d u, \\
& \hat{\rho}(\boldsymbol{k})=(2 \pi)^{-3} \int \rho(\boldsymbol{r}) e^{i \boldsymbol{k} \cdot \boldsymbol{r}} d \boldsymbol{r}
\end{aligned}
$$

are the Fourier transforms of $S(u)$ and $\rho(\boldsymbol{r})$, respectively, and $j_{0}(x)$ is a spherical Bessel function. We note that $\sigma(k)$ is an even function.

If $\rho(\boldsymbol{r})$ is analytic, then $\hat{\rho}(\boldsymbol{k})$ decays exponentially, ${ }^{30}$ and we can substitute the Laurent expansion

$$
\sigma(k)=\sum_{n} \sigma_{n} k^{n}
$$

into Eq. (6), swap the integration and summation, and use the Fourier derivative theorem to obtain

$$
\begin{aligned}
V(\boldsymbol{r}) & =4 \pi \int \sum_{n} \sigma_{n} k^{n} \hat{\rho}(\boldsymbol{k}) e^{i \boldsymbol{k} \cdot \boldsymbol{r}} d \boldsymbol{k} \\
& =4 \pi \sum_{n} \sigma_{n}(i \nabla)^{n} \rho(\boldsymbol{r})=4 \pi \sigma(i \nabla) \rho(\boldsymbol{r}) .
\end{aligned}
$$

Equation (10), a GPE, is the key result of this Communication. It provides an explicit relationship between a density 
$\rho(\boldsymbol{r})$ and its short-range potential $V(\boldsymbol{r})$, assuming that the density is smooth. Furthermore, we note that Eqs. (2) and (10) imply the formal identity

$$
S(u) \equiv 4 \pi \sigma(i \nabla) \delta(\boldsymbol{u}) .
$$

In the unattentuated limit (i.e., $\omega=0$ ), where

$$
S(u)=u^{-1} \Leftrightarrow \sigma(k)=k^{-2},
$$

the GPE reduces to

$$
V(\boldsymbol{r})=-\frac{4 \pi}{\nabla^{2}} \rho(\boldsymbol{r})
$$

which is the familiar Poisson equation. In contrast, if $S(u)$ decays at least exponentially, we can write

$$
\sigma(k)=\int_{0}^{\infty} u^{2} j_{0}(k u) S(u) d u=\mu_{2}-\frac{\mu_{4}}{3 !} k^{2}+\frac{\mu_{6}}{5 !} k^{4}-\cdots,
$$

where $\mu_{j}$ is the $j$ th moment of $S$. If we substitute this into the GPE, we obtain the local expansion

$$
V(\boldsymbol{r})=4 \pi\left[\mu_{2} \rho+\frac{\mu_{4}}{3 !} \nabla^{2} \rho+\frac{\mu_{6}}{5 !} \nabla^{4} \rho+\cdots\right],
$$

which, unlike the Poisson equation [Eq. (13)], expresses the potential directly as a linear combination of the density and its (hyper-) Laplacians.

An immediate corollary of Eq. (15) is that the shortrange self-interaction energy of a density $\rho(\boldsymbol{r})$ can be converted from the usual double integral

$$
E=\frac{1}{2} \iint \rho(\boldsymbol{r}) S(u) \rho\left(\boldsymbol{r}^{\prime}\right) d \boldsymbol{r}^{\prime} d \boldsymbol{r}
$$

into a density functional

$$
E=2 \pi \int\left[\mu_{2} \rho^{2}+\frac{\mu_{4}}{3 !} \rho \nabla^{2} \rho+\frac{\mu_{6}}{5 !} \rho \nabla^{4} \rho+\cdots\right] d \boldsymbol{r}
$$

of the gradient expansion type.

Equation (17) is related to Eq. (C18) in the work of Toulouse et al. ${ }^{8}$, but whereas Toulouse et al. stated that theirs is an asymptotic series, we show below that Eq. (17) is a convergent series if an appropriate attenuator is chosen.

\section{ATTENUATORS AND EXPANSIONS}

The Maclaurin series [Eq. (14)] is easy to find for many $S(u)$ and local expansions for $V(\boldsymbol{r})$ and $E$ immediately follow. For example, the Yukawa attenuator [Eq. (3)] yields

$$
\begin{aligned}
& \sigma_{Y}(k)=\frac{1}{\omega^{2}}-\frac{k^{2}}{\omega^{4}}+\frac{k^{4}}{\omega^{6}}-\cdots, \\
& V_{Y}(\boldsymbol{r})=4 \pi\left[\frac{\rho}{\omega^{2}}+\frac{\nabla^{2} \rho}{\omega^{4}}+\frac{\nabla^{4} \rho}{\omega^{6}}+\cdots\right], \\
& E_{Y}=2 \pi \int\left[\frac{\rho^{2}}{\omega^{2}}+\frac{\rho \nabla^{2} \rho}{\omega^{4}}+\frac{\rho \nabla^{4} \rho}{\omega^{6}}+\cdots\right] d \boldsymbol{r},
\end{aligned}
$$

the Ewald attenuator [Eq. (4)] yields

$$
\begin{aligned}
& \sigma_{E}(k)=\frac{1}{1 !(2 \omega)^{2}}-\frac{k^{2}}{2 !(2 \omega)^{4}}+\frac{k^{4}}{3 !(2 \omega)^{6}}-\cdots, \\
& V_{E}(\boldsymbol{r})=4 \pi\left[\frac{\rho}{1 !(2 \omega)^{2}}+\frac{\nabla^{2} \rho}{2 !(2 \omega)^{4}}+\frac{\nabla^{4} \rho}{3 !(2 \omega)^{6}}+\cdots\right], \\
& E_{E}=2 \pi \int\left[\frac{\rho^{2}}{1 !(2 \omega)^{2}}+\frac{\rho \nabla^{2} \rho}{2 !(2 \omega)^{4}}+\frac{\rho \nabla^{4} \rho}{3 !(2 \omega)^{6}}+\cdots\right] d \boldsymbol{r},
\end{aligned}
$$

and the Heaviside attenuator $[$ Eq. (5)] yields

$$
\begin{aligned}
& \sigma_{H}(k)=\frac{1}{2 ! \omega^{2}}-\frac{k^{2}}{4 ! \omega^{4}}+\frac{k^{4}}{6 ! \omega^{6}}-\cdots, \\
& V_{H}(\boldsymbol{r})=4 \pi\left[\frac{\rho}{2 ! \omega^{2}}+\frac{\nabla^{2} \rho}{4 ! \omega^{4}}+\frac{\nabla^{4} \rho}{6 ! \omega^{6}}+\cdots\right], \\
& E_{H}=2 \pi \int\left[\frac{\rho^{2}}{2 ! \omega^{2}}+\frac{\rho \nabla^{2} \rho}{4 ! \omega^{4}}+\frac{\rho \nabla^{4} \rho}{6 ! \omega^{6}}+\cdots\right] d \boldsymbol{r} .
\end{aligned}
$$

Each of formulas (20), (23), and (26) expresses the short-range self-interaction energy as a local density functional and, in cases where the function $S(u)$ is very short ranged compared with the density $\rho(\boldsymbol{r})$, they offer a natural alternative to the conventional double integral [Eq. (16)].

\section{RANGE OF APPLICABILITY}

Although the expansions above for the potential and energy are formally exact, they are not guaranteed to converge in all situations.

First, they will systematically diverge if we unwisely choose a function $S(u)$ that is long ranged compared with the density. Consider, for example, the Gaussian density

$$
\rho(\boldsymbol{r})=(2 / \pi)^{3 / 2} \exp \left(-2 r^{2}\right) .
$$

It is not difficult to show that

$$
\int \rho \nabla^{2 n} \rho d \boldsymbol{r}=\frac{(-1)^{n}}{2 \pi^{3 / 2}} \frac{(2 n+2) !}{(n+1) !},
$$

and the Yukawa, Ewald, and Heaviside expansions are

$$
\begin{aligned}
& E_{Y}=\frac{1}{\sqrt{\pi}}\left[\frac{2 !}{1 ! \omega^{2}}-\frac{4 !}{2 ! \omega^{4}}+\frac{6 !}{3 ! \omega^{6}}-\cdots\right], \\
& E_{E}=\frac{1}{\pi}\left[\frac{\Gamma(3 / 2)}{1 ! \omega^{2}}-\frac{\Gamma(5 / 2)}{2 ! \omega^{4}}+\frac{\Gamma(7 / 2)}{3 ! \omega^{6}}-\cdots\right], \\
& E_{H}=\frac{1}{\sqrt{\pi}}\left[\frac{1}{1 ! \omega^{2}}-\frac{1}{2 ! \omega^{4}}+\frac{1}{3 ! \omega^{6}}-\cdots\right],
\end{aligned}
$$

respectively. Because the Yukawa attenuator decays only exponentially, it is longer ranged than the density and its series diverges for all $\omega$. For similar reasons, the Ewald series is convergent if, and only if, $\omega \geqslant 1$. The Heaviside attenuator, in contrast, has compact support, and its series therefore converges for any value of $\omega$. 
Second, if $\rho(\boldsymbol{r})$ contains singularities, the swap of integration and summation in Eq. (10) cannot be justified and expansions (15) and (17) are no longer exact. This is illustrated by the exact hydrogen atom density

$$
\rho(\boldsymbol{r})=(1 / \pi) \exp (-2 r),
$$

which has a cusp singularity at $r=0$ and for which

$$
2 \pi \int \rho \nabla^{2 n} \rho d \boldsymbol{r}=(1-2 n) 2^{2 n-2} .
$$

Using the Heaviside attenuator [Eq. (5)], the true short-range energy [Eq. (16)] can be shown to be

$$
E_{H}=\frac{5}{16}-\left(\frac{5}{16}+\frac{5}{8 \omega}+\frac{1}{2 \omega^{2}}+\frac{1}{6 \omega^{3}}\right) \exp \left(-\frac{2}{\omega}\right),
$$

which yields the correct (convergent) series expansion

$$
E_{H}=\frac{1}{8 \omega^{2}}-\frac{1}{24 \omega^{4}}+\frac{1}{36 \omega^{6}}-\frac{8}{315 \omega^{7}}+\frac{1}{72 \omega^{8}}-\cdots .
$$

In contrast, Eq. (26) gives the incorrect expansion

$$
E_{H}=\frac{1}{8 \omega^{2}}-\frac{1}{24 \omega^{4}}-\frac{1}{60 \omega^{6}}-\frac{1}{504 \omega^{8}}-\frac{1}{8100 \omega^{10}}-\cdots .
$$

Because the cusp in $\rho(\boldsymbol{r})$ produces an $O\left(\omega^{-6}\right)$ error, the GPE is probably less useful for such densities.

Of course, approximate (cuspless) hydrogen atom densities present no such problems. If the Heaviside attenuator with $\omega=1$ is applied to the HF/STO-3G density of the H atom, the sum of the first 16 terms of Eq. (17) is correct to one part in $10^{8}$.

\section{LARGER SYSTEMS}

We have examined sums of $K$ identical Gaussians

$$
g_{a}(\boldsymbol{r})=(2 / \pi)^{3 / 2} \exp \left(-2\left|\boldsymbol{r}-\boldsymbol{R}_{a}\right|^{2}\right)
$$

arranged in linear $(d=1)$, square $(d=2)$, and cubic $(d=3)$ lattices, each with lattice constant $c$.

To find the short-range self-interaction energy of such densities via Eq. (17), one needs the one-electron integral

$$
\int g_{a}(\boldsymbol{r}) \nabla^{2 n} g_{b}(\boldsymbol{r}) d \boldsymbol{r}=\frac{H_{2 n+1}(R)}{2 \pi^{3 / 2} R} \exp \left(-R^{2}\right)
$$

between two Gaussians with $\left|\boldsymbol{R}_{a}-\boldsymbol{R}_{b}\right|=R$, where $H_{n}(x)$ is a Hermite polynomial. We note that Eq. (38) reduces to Eq. (28) when $R=0$.

For comparison, the energy can also be found via Eq. (16) using the double integral

$$
I(R)=\iint g_{a}(\boldsymbol{r}) S(u) g_{b}\left(\boldsymbol{r}^{\prime}\right) d \boldsymbol{r}^{\prime} d \boldsymbol{r} .
$$

The standard Fourier transform approach ${ }^{22}$ yields

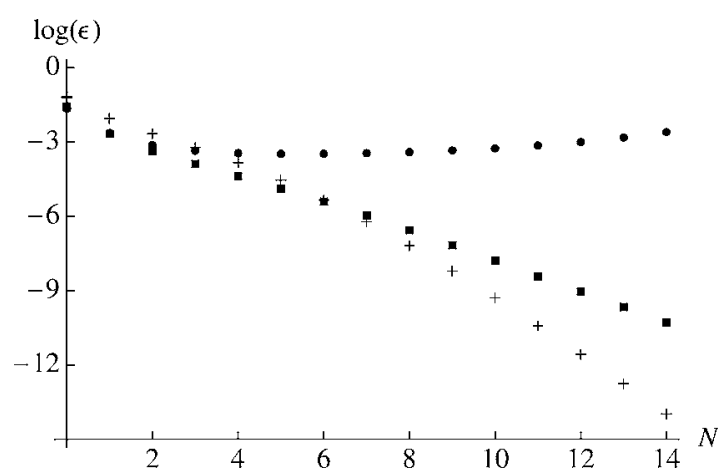

FIG. 1. Variation of $\epsilon$ with $N$ for $S_{Y}(\bullet, \omega=6.0), S_{E}(\boldsymbol{\square}, \omega=2.0)$, and $S_{H}(+, \omega=1.0)$ using $K=125, c=1.0$, and $d=3$.

$$
I(R)=\frac{2}{\pi} \int_{0}^{\infty} k^{2} j_{0}(k R) \exp \left(-k^{2} / 4\right) \sigma(k) d k
$$

which can be evaluated in terms of the error function for each of the attenuators in Eqs. (3)-(5).

Truncation of Eq. (17) after a finite number $N+1$ of terms yields an approximation $E_{\text {approx }}$ whose relative error $\epsilon$ depends on the attenuator $S(u)$, the attenuation strength $\omega$, the number $K$ of Gaussians, the lattice constant $c$, and the dimensionality $d$ of the lattice.

Figure 1 shows the variation of $\epsilon$ with $N$ for the three attenuators for fixed $\omega, K, c$, and $d$. For Gaussian densities, the Yukawa expansion [Eq. (20)] is an asymptotic series which, after initially converging, eventually diverges. In contrast, the Ewald expansion [Eq. (23)] exhibits exponential convergence and the Heaviside expansion [Eq. (26)] converges superexponentially. Evidently, the attenuators' convergence behaviors for 125 Gaussians follow exactly the patterns observed in Eqs. (29)-(31) for a single Gaussian.

Figure 2 shows the variation of $\epsilon$ with $c$ for three attenuation rates for fixed $S(u), K, d$, and $N$. (We note that varying $c$ with a fixed Gaussian exponent is equivalent to varying the exponent with fixed $c$.) At the two extremes ( $c=0$ where the density is a single Gaussian and $c=\infty$ where it is $K$ noninteracting Gaussians), the same relative error is obtained. For $c$ values comparable to the inverse square root of the Gaussian exponent in Eq. (37), the accuracy improves somewhat because the density is approximately uniform.

Figure 3 shows the variation of $\epsilon$ with $K$ for the three

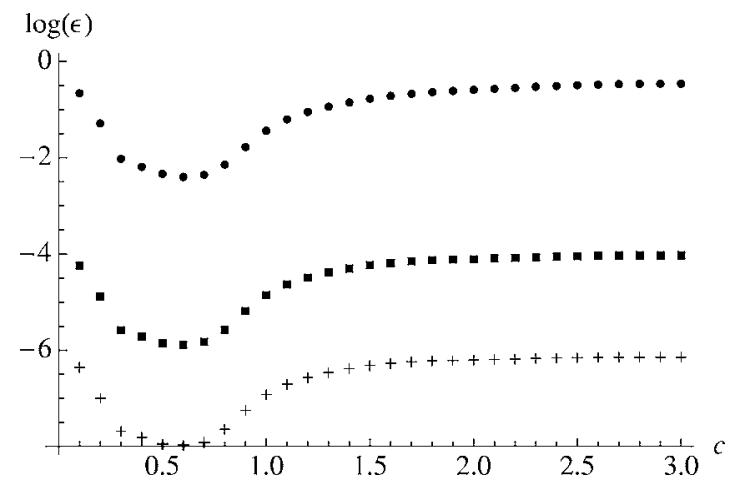

FIG. 2. Variation of $\epsilon$ with $c$ for $\omega=1.0(\bigcirc), 2.0(\square)$, and $3.0(+)$ using $S_{E}$, $K=125, d=3$, and $N=5$. 


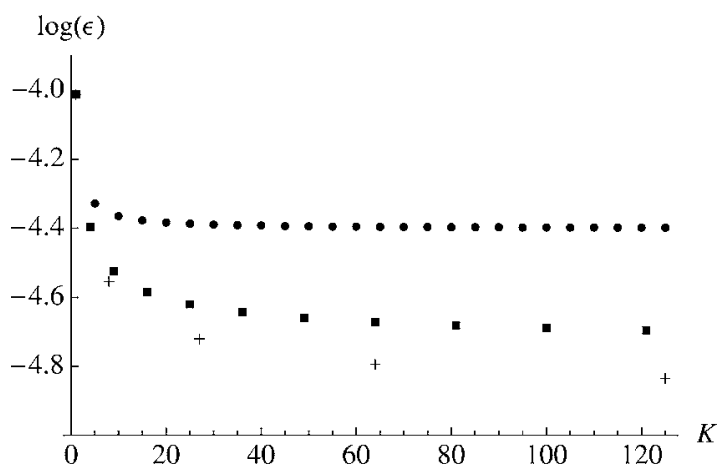

FIG. 3. Variation of $\epsilon$ with $K$ for $d=1(\bullet), 2(\square)$, and $3(+)$ using $S_{E}, \omega$ $=2.0, c=1.0$, and $N=5$.

lattices for fixed $S(u), c$, and $N$. The relative error decreases toward a limiting value as $K$ increases, which is consistent with the fact that the short-range energy is an extensive quantity. The limiting error is reached most rapidly in the low-dimensional systems, where the number of neighboring Gaussians is small.

\section{CONCLUDING REMARKS}

We have introduced a GPE that relates a smooth density $\rho(\mathbf{r})$ to its short-range Newtonian potential $V(\mathbf{r})$. In cases where the attenuation is exponential or faster, the GPE provides a local mapping between $\rho(\mathbf{r})$ and $V(\mathbf{r})$ and can be solved without integration. Furthermore, provided that the attenuation is short ranged compared with the density, the series for $V(\mathbf{r})$ is convergent.

The GPE will be useful in next-generation electronic structure methods that require the short-range part of the Coulomb potential because it is simpler than previous approaches and its cost is comparable to the calculation of the density itself. In nonconducting systems (where the density matrix is sparse), this cost will scale linearly with system size. It may also be helpful in constructing improved short-range density functionals in the context of the various long-range-corrected density functional theory methods. ${ }^{5,8-10,16-18,20}$

\section{ACKNOWLEDGMENTS}

S.A.V. thanks ANU's Research School of Chemistry for a postdoctoral fellowship.

${ }^{1}$ I. Panas, Chem. Phys. Lett. 245, 171 (1995).

${ }^{2}$ A. Savin, Recent Developments of Modern Density Functional Theory (Elsevier, Amsterdam, 1996).

${ }^{3}$ J. P. Dombroski, S. W. Taylor, and P. M. W. Gill, J. Phys. Chem. 100, 6272 (1996).

${ }^{4}$ P. M. W. Gill, R. D. Adamson, and J. A. Pople, Mol. Phys. 88, 1005 (1996).

${ }^{5}$ H. Iikura, T. Tsuneda, T. Yanai, and K. Hirao, J. Chem. Phys. 115, 3540 (2001).

${ }^{6}$ I. Sirbu and H. F. King, J. Chem. Phys. 117, 6411 (2002).

${ }^{7}$ J. Heyd, G. E. Scuseria, and M. Ernzerhof, J. Chem. Phys. 118, 8207 (2003).

${ }^{8}$ J. Toulouse, F. Colonna, and A. Savin, Phys. Rev. A 70, 062505 (2004).

${ }^{9}$ Y. Tawada, T. Tsuneda, S. Yanagisawa, T. Yanai, and K. Hirao, J. Chem. Phys. 120, 8425 (2004).

${ }^{10}$ T. Yanai, D. P. Tew, and N. C. Handy, Chem. Phys. Lett. 393, 51 (2004).

${ }^{11}$ R. Baer and D. Neuhauser, Phys. Rev. Lett. 94, 043002 (2005).

${ }^{12}$ I. C. Gerber and J. G. Angyan, Chem. Phys. Lett. 415, 100 (2005).

${ }^{13}$ J. Toulouse and A. Savin, J. Mol. Struct.: THEOCHEM 762, 147 (2006).

${ }^{14}$ J. G. Angyan, I. Gerber, and M. Marsman, J. Phys. A 39, 8613 (2006).

${ }^{15}$ O. A. Vydrov, J. Heyd, A. V. Krukau, and G. E. Scuseria, J. Chem. Phys. 125, 074106 (2006).

${ }^{16}$ M. J. G. Peach, A. J. Cohen, and D. J. Tozer, Phys. Chem. Chem. Phys. 8, 4543 (2006).

${ }^{17}$ T. Sato, T. Tsuneda, and K. Hirao, J. Chem. Phys. 126, 234114 (2007).

${ }^{18}$ J. W. Song, S. Tokura, T. Sato, M. A. Watson, and K. Hirao, J. Chem. Phys. 127, 154109 (2007).

${ }^{19}$ A. D. Dutoi and M. Head-Gordon, J. Phys. Chem. A 112, 2110 (2008)

${ }^{20}$ J. D. Chai and M. Head-Gordon, J. Chem. Phys. 128, 084106 (2008).

${ }^{21}$ R. D. Adamson, J. P. Dombroski, and P. M. W. Gill, J. Comput. Chem. 20, 921 (1999).

${ }^{22}$ P. M. W. Gill, Adv. Quantum Chem. 25, 141 (1994).

${ }^{23}$ P. M. W. Gill and R. D. Adamson, Chem. Phys. Lett. 261, 105 (1996).

${ }^{24}$ H. Yukawa, Proc. Phys. Math. Soc. Jpn. 17, 48 (1935).

${ }^{25}$ P. P. Ewald, Ann. Phys. 64, 253 (1921).

${ }^{26}$ R. D. Adamson, J. P. Dombroski, and P. M. W. Gill, Chem. Phys. Lett. 254, 329 (1996).

${ }^{27}$ S. J. Chakravorty and E. Clementi, Phys. Rev. A 39, 2290 (1989).

${ }^{28}$ A. W. Overhauser, Can. J. Phys. 73, 683 (1995).

${ }^{29}$ A. M. Lee, S. W. Taylor, J. P. Dombroski, and P. M. W. Gill, Phys. Rev. A 55, 3233 (1997).

${ }^{30}$ J. P. Boyd, Chebyshev and Fourier Spectral Methods, 2nd ed. (Dover, New York, 2000) 